\title{
Trailing-edge noise prediction using synthetic turbulence and acoustic perturbation equations in a hybrid methodology
}

\author{
A.H. Kadar* , P. Martinez-Lera ${ }^{\dagger}$ \\ Michel Tournour $\ddagger$ \\ Siemens Industry Software NV, Interleuvenlaan 68, 3001 Leuven, Belgium \\ C. Schram ${ }^{\S}$ \\ von Karman Institute for Fluid Dynamics, Chaussee de Waterloo 72, 1640 Rhode-St-Genese, Belgium \\ W. De Roeck ${ }^{\pi}$, W. Desmet $\|$ \\ KU Leuven, Department of Mechanical Engineering, Celestijnenlaan 300, 3001 Leuven, Belgium
}

\begin{abstract}
In this work a hybrid RANS/CAA methodology is used to predict trailing-edge noise of a controlled-diffusion airfoil. A time domain stochastic solver based on the Random Particle Mesh method introduced by Ewert et al. is used to generate turbulent fluctuations in 2-D that accurately reproduce statistical and mean-flow features provided by steady RANS. Acoustic Perturbation Equations coupled with the stochastic solver are solved in time domain, using a vortex sound source term defined in terms of stochastically generated turbulent velocity fluctuations. The far-field sound pressure level obtained using Kirchhoff extrapolation method followed by 2-D to 3-D correction taking into account the finite span of the airfoil is compared with the available experimental data. The numerical results are found to be in good agreement for the frequencies for which experimental data is available. The results indicate the suitability of the 2-D RANS/CAA approach for trailing-edge noise prediction from airfoils with uniform cross-section in the spanwise direction.
\end{abstract}

\section{Introduction}

Trailing-edge noise constitutes a primary source of the noise emitted by low-speed cooling fans in automotive applications. ${ }^{1}$ Trailing-edge noise results from the scattering of the boundary layer disturbances into acoustic waves at the trailing-edge of an airfoil surface and is computationally expensive to account for due to its broadband nature. Attempts have been made in the past to predict airfoil trailing-edge noise using CAA techniques with aerodynamic noise sources obtained using high-fidelity CFD (LES or DES). For instance a first LES of the flow over the controlled-diffusion airfoil derived from the Valeo automotive cooling module was performed by Wang et al. ${ }^{2}$ A structured mesh with 5.1 million nodes was used to yield stable and accurate flow solutions. Subsequent attempts to reduce the computational cost includes LES on un-structured grid ${ }^{3}$ and structured grid. ${ }^{4}$ It is found that the hybrid CAA techniques with flow input from LES can provide accurate noise predictions. However, LES remains computationally expensive for many industrial applications.

An alternative to performing LES for source generation is by stochastically generating turbulent velocity fluctuations which can be used to define acoustic source terms in an aeroacoustic analogy. A stochastic method able to synthesis turbulent velocity fluctuations using only statistical and mean-flow features provided

\footnotetext{
*Research Engineer.

$\dagger$ Senior Research Engineer.

$¥$ Product Development Manager

$\S$ Associate Professor.

I Assistant Professor.

"Full Professor.
} 
by low-cost steady RANS simulations was introduced by Ewert et al. ${ }^{5-9}$ The application of the method for broadband trailing-edge noise prediction has been demonstrated in previous works. ${ }^{10}$

In this work trailing-edge noise from a controlled-diffusion airfoil is predicted using synthetic turbulence and acoustic perturbation equations in a hybrid methodology. The outline of the paper is as follows: In section II the underlying theory of the stochastic method used for generating isotropic and locally homogeneous turbulence in 2-D is briefly explained. In section III.A the results from the 2-D RANS computation of the controlled-diffusion airfoil are validated with the available experimental data. In section III.B the input parameters required for the stochastic reconstruction method are stated. The quality of the synthetic turbulence generated by the stochastic method is assessed by comparing the statistics of the reconstructed fluctuations with the input RANS statistics. In section III.C the acoustic perturbation equations are presented. The obtained velocity fluctuations are used to define the vortex sound source terms for the acoustic perturbation equations. The acoustic perturbation equations in the propagation domain are solved by a quadrature-free discontinuous Galerkin solver. ${ }^{11}$ Finally in the results section the far-field sound pressure level obtained after Kirchhoff extrapolation method(section III.D) followed by 2-D to 3-D correction(section III.E) taking into account the finite span of the airfoil is compared with the available experimental data.

\section{Synthetic turbulence generation}

Aeroacoustic analogy's governing the propagation of flow generated sound can be formally expressed in the form

$$
\Gamma p^{\prime}(\vec{x}, t)=q_{s}(\vec{x}, t)
$$

where $\Gamma$ is the acoustic propagation operator, $p^{\prime}$ is the acoustic pressure fluctuation and $q_{s}$ is the source term. The far-field noise caused by the source term $q_{s}$ is unambiguously defined by the two-point cross-correlation of the source (Ewert et. al. ${ }^{9}$ ) given by:

$$
R^{q_{s}}\left(\overrightarrow{x_{1}}, \overrightarrow{x_{2}}, t_{1}, t_{2}\right)=\left\langle q_{s}\left(\overrightarrow{x_{1}}, t_{1}\right) q_{s}\left(\overrightarrow{x_{2}}, t_{2}\right)\right\rangle .
$$

A filter based method able to synthesize turbulent velocity fluctuations with the desired two-point crosscorrelation has been developed by Ewert et. al. ${ }^{5-9}$ and is known as Random Particle-Mesh (RPM) method. The method can be applied in conjunction with an acoustic analogy when the source term $q_{s}$ can be expressed as a linear function of velocity fluctuations.

Building upon the work of Ewert et. al. a purely Lagrangian approach to the RPM method was proposed by Dieste. ${ }^{12}$ The method is able to generate locally homogeneous, isotropic and evolving turbulence in both 2-D and 3-D. In the present work the RPM solver ${ }^{11}$ based on the Lagrangian approach of Dieste ${ }^{12}$ is used in conjunction with vortex sound theory to predict trailing-edge noise.

In the framework of the Random Particle-Mesh method the turbulent fluctuations are stochastically generated by spatially filtering convected white noise. In Dieste's approach the discrete realization of the convected white-noise is by means of Lagrangian particles distributed over the source domain and convected by the RANS mean flow. The method requires as input statistical properties of turbulence such as the distribution of turbulence kinetic energy, integral length and time scales of turbulence. In addition the spatial filter is derived by prescribing a model for the energy spectrum of turbulence.

The velocity fluctuations are obtained by spatially filtering convected white-noise as follows (for detailed derivation refer to Dieste et. al. ${ }^{12}$ )

$$
\vec{u}(\vec{x}, t)=\sum_{n=1}^{N} \vec{G}\left(r_{n}, k\left(\overrightarrow{y_{n}}\right), \Lambda\left(\overrightarrow{y_{n}}\right)\right) W_{n}(t, \tau) .
$$

where $\vec{G}=\nabla \times(0,0, G)$ and $G$ is the prescribed Gaussian filter in space derived from Gaussian energy spectrum in the wave number domain for locally homogeneous and isotropic turbulence.

$$
G(r)=\sqrt{\frac{2 k}{\pi}} e^{\frac{-\pi r^{2}}{2 \Lambda^{2}}}
$$


The filter $G$ is a function of the turbulence kinetic energy $(k)$ and integral length $\operatorname{scale}(\Lambda)$. The velocity fluctuation $\vec{u}(\vec{x}, t)$ at a desired location $\vec{x}$ is obtained by summing over all the $N$ random particles in the source domain on which the white noise field $W_{n}$ is defined. The strength of the random particle at each time is obtained using the Langevin equation in the Lagrangian frame given by:

$$
\frac{\partial W_{n}(t)}{\partial t}=\frac{-1}{\tau} W_{n}(t)+\sqrt{\frac{2}{\tau}} \varsigma_{n}(t)
$$

where $W_{n}$ and $\varsigma_{n}$ are zero-mean Gaussian distribution white noise fields with variance determined by the density of particles. The Langevin equation prescribes evolving turbulence with exponential time-decorrelation. The equation is numerically integrated using higher order Runge-Kutta time integration schemes.

\section{Application: Trailing-edge noise from controlled-diffusion airfoil}

The airfoil geometry considered in the present work is the controlled-diffusion(CD) airfoil derived from the Valeo automotive cooling module. controlled-diffusion airfoils are a class of cambered airfoils that control the diffusion of velocity over the surface increasing the amount of laminar flow in relation to turbulent flow. This delays the flow separation before the trailing edge providing higher aerodynamic loading. The profile of the CD airfoil, corresponding to the Valeo cooling blade profile at mid-span is shown in Figure 1. The airfoil has a chord length $\left(C_{L}\right)$ of $0.1356 \mathrm{~m}$ and a span length(L) of $0.3 \mathrm{~m}$.

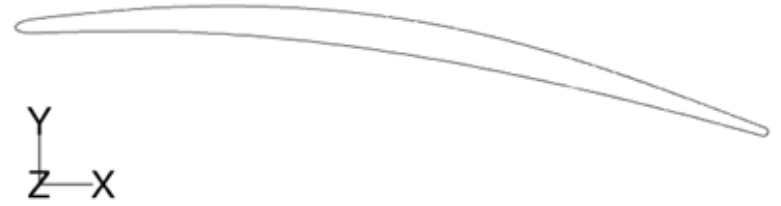

Figure 1. Schematic of the CD airfoil.

The CD airfoil has been thoroughly investigated experimentally. The experimental data were collected at two separate facilities. The large anechoic wind tunnel of the Ecole Centrale de Lyon (ECL) was initially used for the wall-pressure measurements. ${ }^{13}$ Additional experiments using hot-wire anemometry were performed at the Turbulent Shear Flow Laboratory(TSFL) at Michigan State University ${ }^{14}$ reproducing the flow conditions found in the ECL wind tunnel.

\section{A. 2-D steady RANS simulation of the controlled-diffusion airfoil}

In the present work the hybrid methodology to predict aerodynamic noise is applied to the CD airfoil at an angle of attack $\alpha=8^{\circ}$. The flow Reynolds number $R e_{c}=1.6 \times 10^{5}$ is based on the free stream velocity $U_{\infty}=16 \mathrm{~m} / \mathrm{s}$, airfoil chord length $\left(C_{L}\right)$ and kinematic viscosity of air $\nu_{a i r}=1.356 \times 10^{-5} \mathrm{~m}^{2} / \mathrm{s}$. The RANS domain and mesh are shown in Figure 2. The size of the computational domain is $4 C_{L}$ in the stream wise direction and $2.5 C_{L}$ in the transverse direction. The domain is discretized using a block-structured mesh with 476,625 predominantly hexahedral cells distributed across 5 levels of refinement. The mesh generated using snappyHexMesh utility approximately conforms to the surface by iteratively refining a starting coarse background mesh. 

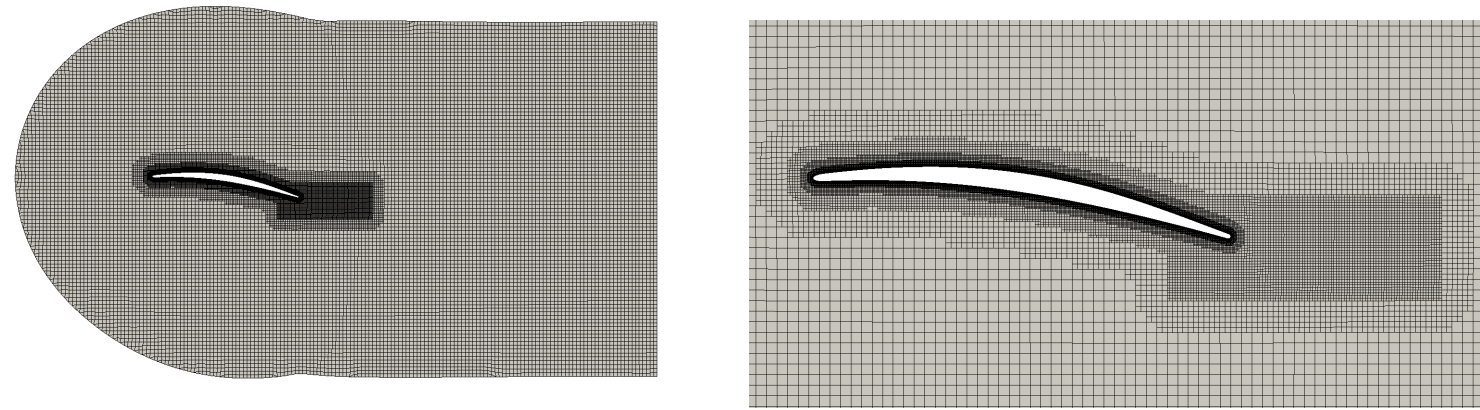

Figure 2. a) 2-D RANS mesh. b) Mesh refinement in the boundary layer and in the wake of the airfoil.

Incompressible 2-D RANS computations using the $k-\omega$ SST RANS turbulence model are performed using the Finite Volume solver OpenFOAM 3.0. ${ }^{15}$ The discretization schemes used are second-order accurate in space and time. The flow is non-dimensionalized using airfoil chord length $\left(C_{L}\right)$ and free stream velocity $\left(U_{\infty}\right)$. In order to take into account the jet deflection that is observed in the experiments the velocity boundary conditions at the inlet are extracted from a RANS computation performed on a larger domain including the airfoil, nozzle and the jet. ${ }^{4}$ A no-slip boundary condition on the airfoil surface and a pressure outlet boundary condition at the exit are prescribed. The turbulence kinetic energy $(k)$ at the inlet is calculated from the turbulence intensity $I=0.7 \%$ corresponding to the experiments as follows:

$$
k=\frac{3}{2}\left(U_{\infty} I\right)^{2} .
$$

The turbulence specific dissipation $\operatorname{rate}\left(\omega_{t}\right)$ at the inlet is calculated from the mixing length $l_{m}\left(\sim C_{L}\right)$ as follows:

$$
\omega_{t}=\frac{k^{1 / 2}}{C_{\mu}{ }^{1 / 4} l_{m}} .
$$

The streamline plot of the non-dimensional velocity field is shown in Figure 3. The distribution of turbulence kinetic energy close to the trailing-edge and in the wake of the airfoil is shown in Figure 4.

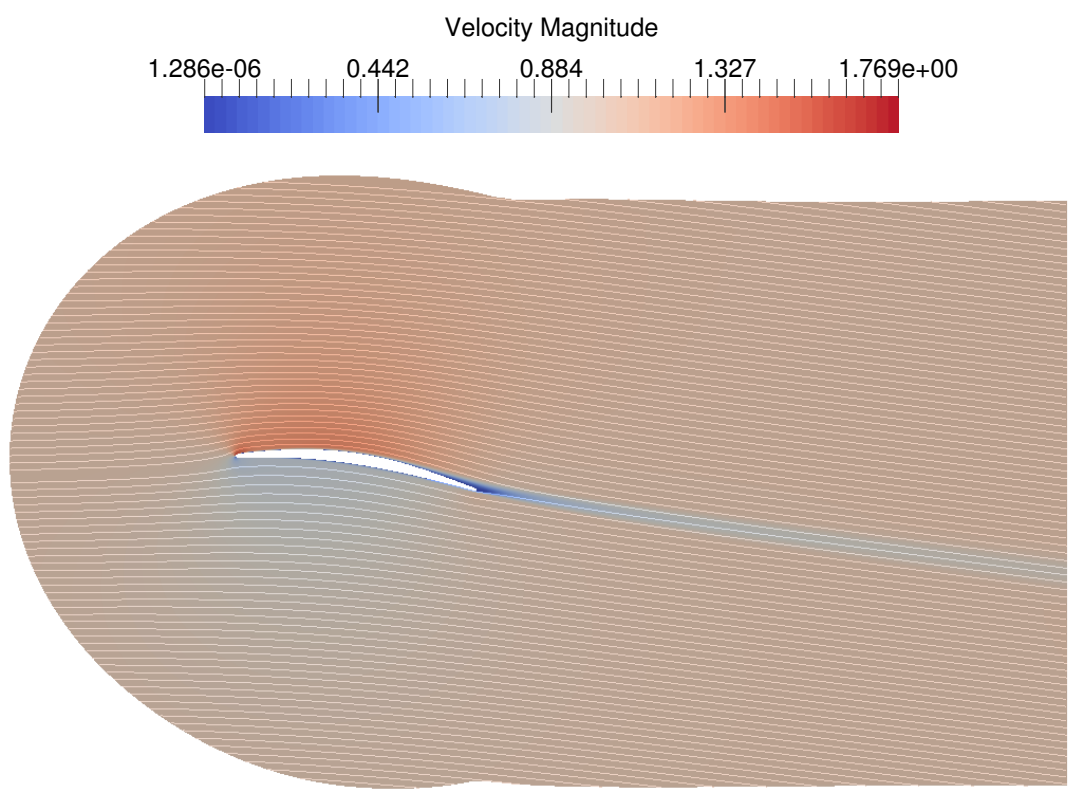

Figure 3. Flow streamlines around the CD airfoil computed using the $k-\omega$ SST RANS turbulence model. 


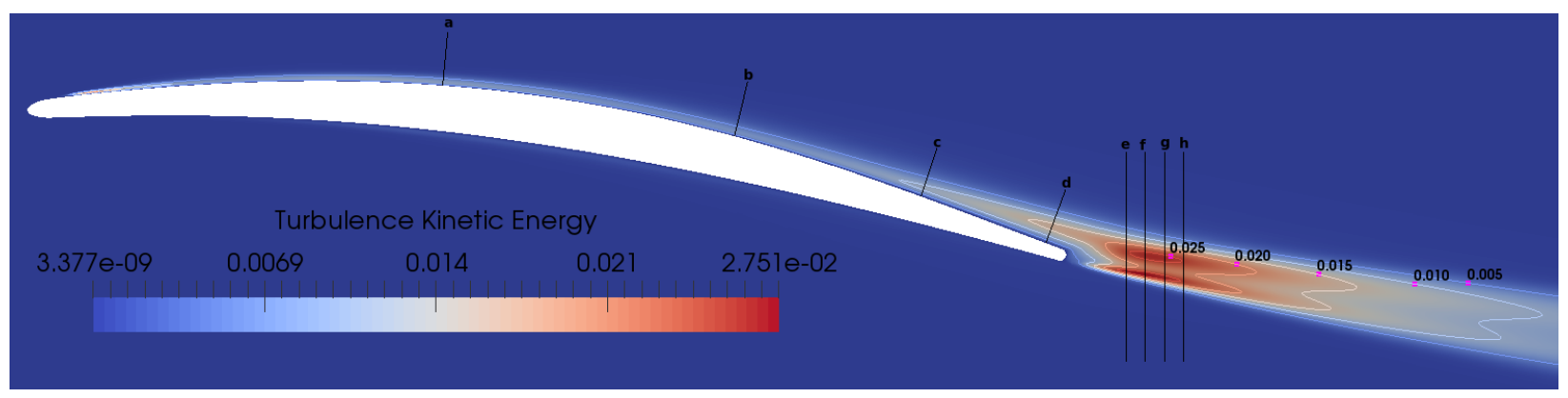

Figure 4. Contours of turbulence kinetic energy close to the trailing-edge and in the wake of the airfoil. The RANS data is extracted along the lines a) $\left.\left.\left.x_{c} / C_{L}=\mathbf{- 0 . 6}, \mathbf{b}\right) x_{c} / C_{L}=\mathbf{- 0 . 3 2}, \mathbf{c}\right) x_{c} / C_{L}=\mathbf{- 0 . 1 4}, \mathbf{d}\right) x_{c} / C_{L}=-0.02$, e) $\left.\left.\left.x / C_{L}=0.0574, \mathbf{f}\right) x / C_{L}=0.0753, \mathrm{~g}\right) x / C_{L}=0.0940, \mathrm{~h}\right) x / C_{L}=0.1126$ and compared with experimental data. Here $x_{c}=x / \cos (\alpha)$ is the chordwise coordinate used to represent data along the blade chord, where $\alpha$ is the angle of attack.

The mean pressure on the airfoil surface characterized by the pressure coefficient $-C_{p}$ is shown in Figure 5. The results are in good agreement compared with the measurements by Moreau \& Roger ${ }^{16}$ except in the leading-edge re-circulation region where small differences are observed. This is understood to be due to the limitation of the RANS model which solves equations governing fully turbulent flows without any transition model. It is therefore not able to correctly predict the transition from laminar to turbulent boundary layer near the leading-edge on the suction side triggered by an unsteady laminar separation.

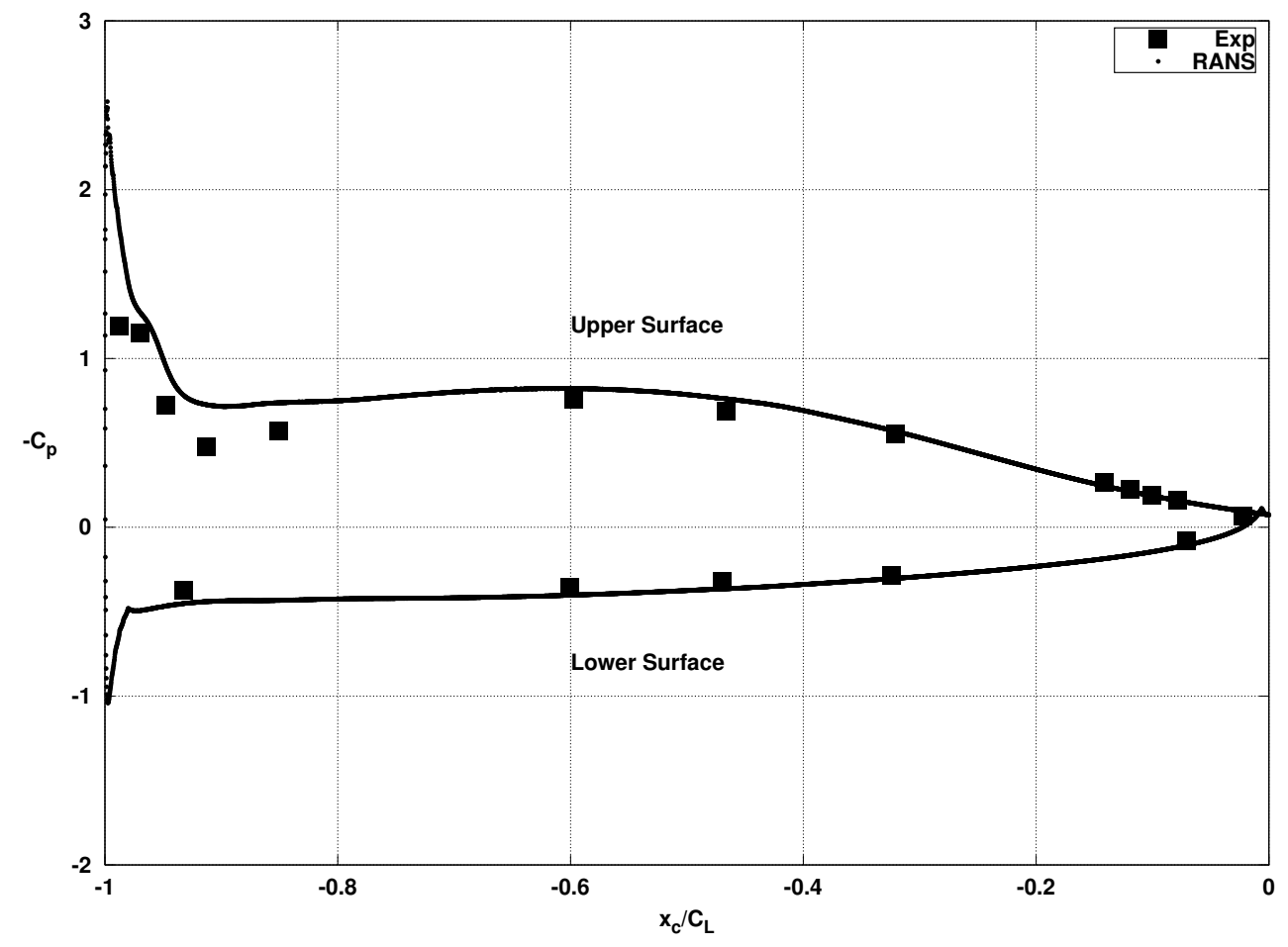

Figure 5. Mean wall-pressure coefficient $\left(C_{p}\right)$ along airfoil surface.

The boundary-layer turbulence intensity profiles from RANS computation are extracted along lines perpendicular to the wall at stations (a), (b), (c) and (d) as shown in Figure 4. The numerical results are compared with experimental data in Figure 6 . The growth of boundary layer on approaching the trailingedge region is evident from both numerical and experimental data. The turbulence intensity for the last station (d) depicts a more broader profile as compared to a narrow spike very close to the wall for the first station (a). The results for the second and third stations are not in very good agreement with experimental 
data, the primary reason is again attributed to the limitation of RANS model which fails to capture the correct transition from laminar to turbulent boundary layer near the leading-edge resulting into errors downstream. Nevertheless at the last station (d) close to the trailing-edge the results are in reasonable agreement with experimental data. Since in this work the focus is to compute the noise due to trailing-edge turbulence the present computations are deemed sufficiently accurate to capture the noise sources.

(a)

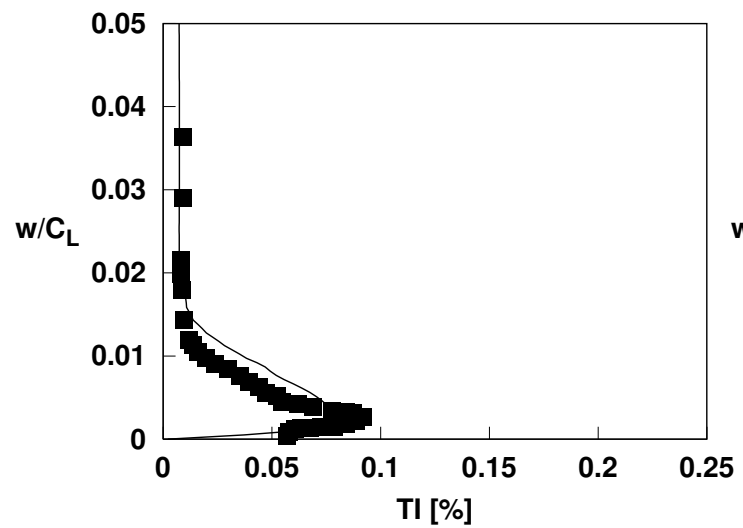

(c)

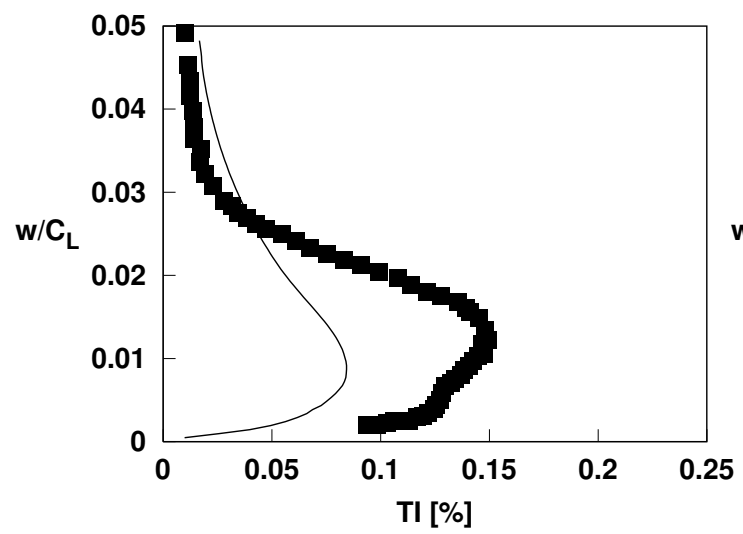

(b)

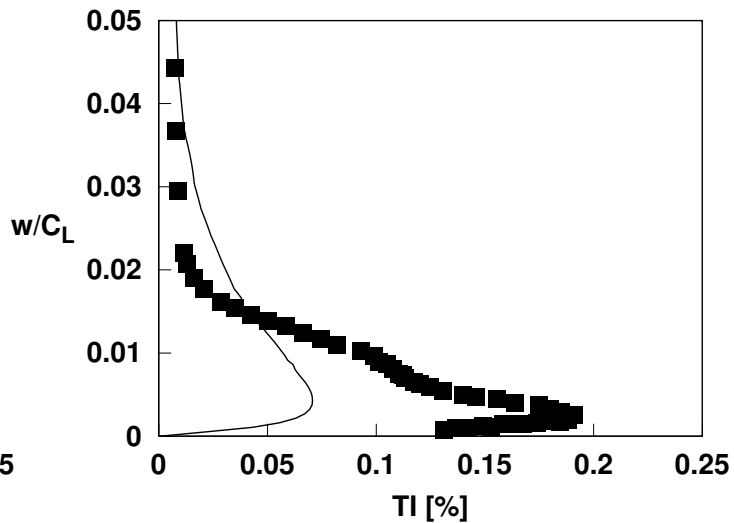

(d)

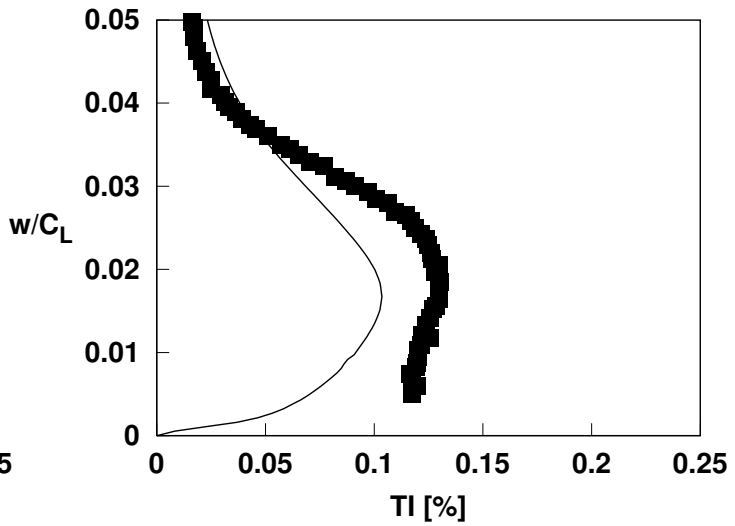

Figure 6. Boundary layer turbulence intensity profiles at stations (a), (b), (c) and (d) as shown in Figure 4. Here $w / C_{L}$ is the perpendicular distance from the airfoil wall normalized by the chord length.

The mean streamwise and crosswise velocity at stations (e), (f), (g) and (h) are compared with experimental data recorded by Moreau et al. ${ }^{14}$ in Figure 7 . For the same stations the RMS streamwise and crosswise fluctuations are compared with experimental data in Figure 8. The RANS computations present a good qualitative agreement with experimental data. It is observed that the maximum peak for both the mean and RMS are shifted towards the suction side compared to experiments and the misalignment is increased on moving away from the trailing-edge as observed for the last station (h). Nevertheless as long as close to the trailing-edge the results are in reasonable agreement with experiments, the numerical computations are acceptable for acoustic computation. 

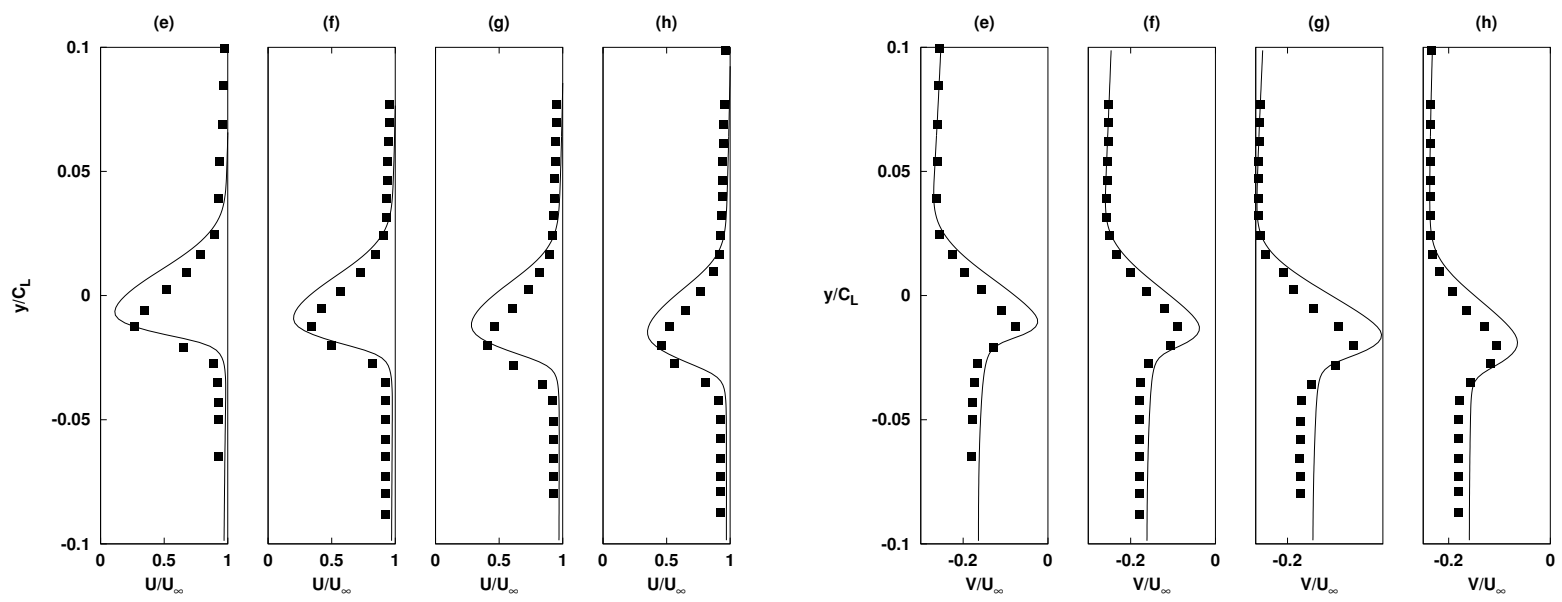

Figure 7. Wake velocity data for the normalized mean streamwise $U / U_{\infty}$ (left) and crosswise $\left(V / U_{\infty}\right)$ (right) components, at stations (e), (f), (g) and (h) as shown in Figure 4.
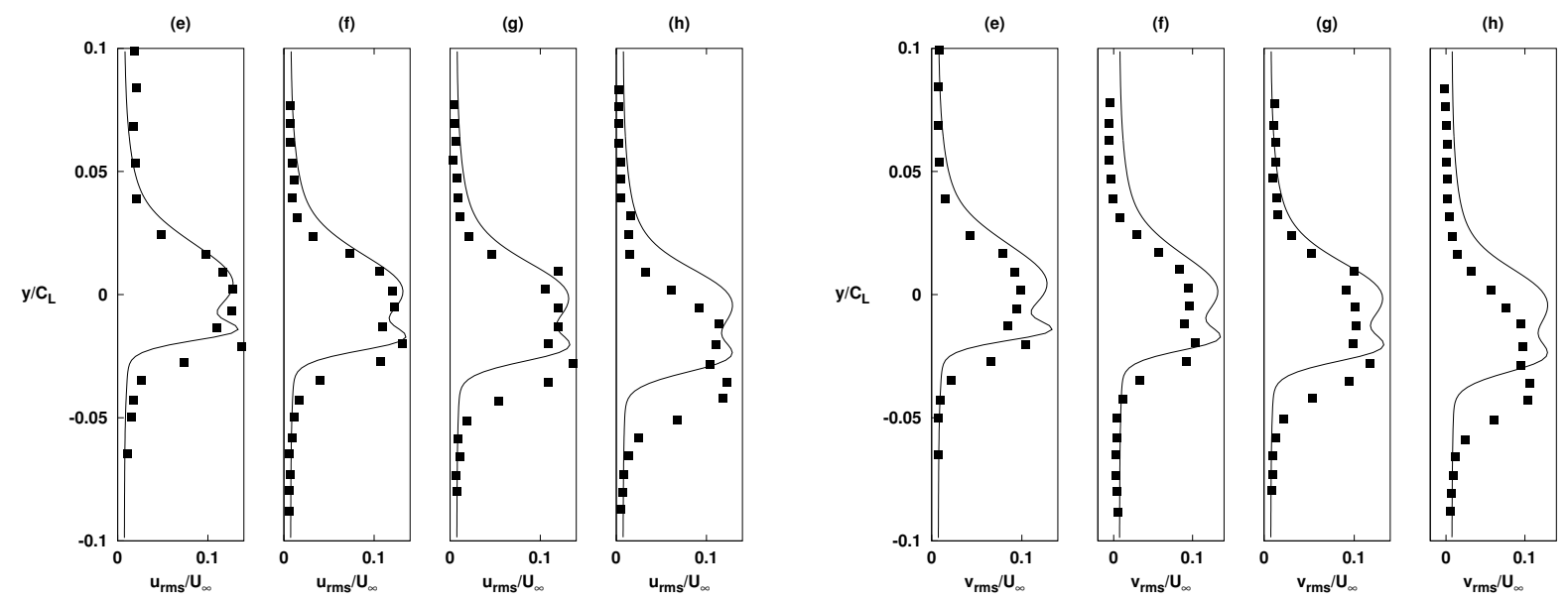

Figure 8. Wake velocity data for the normalized RMS streamwise $\bar{u}^{\prime} / U_{\infty}$ (left) and crosswise $\overline{v^{\prime}} / U_{\infty}$ (right) fluctuations, at stations (e), (f), (g) and (h) as shown in Figure 4.

\section{B. Stochastic reconstruction of turbulent fluctuations in 2-D}

The sources of sound are localized close to the trailing edge and in the wake where the turbulence kinetic energy is high. The RPM domain in 2-D is therefore restricted to a rectangular box including the trailingedge and the airfoil wake as shown in Figure 9. The RANS flow domain is rotated by the angle of attack $\alpha=8^{\circ}$ to facilitate the computation of space and time correlations along the streamline aligned with the $\mathrm{x}$-Axis as shown in Figure 10.a. The RANS simulation results are further non-dimensionalized with the speed of sound $c_{s}=340 \mathrm{~m} / \mathrm{s}$. This allows generating the acoustic source terms non-dimensionalized with the speed of sound which is the adopted convention of the discontinuous Galerkin solver ${ }^{17}$ used for acoustic propagation in the present work. Henceforth, all the subsequent physical quantities presented in the paper should be considered to be non-dimensionalized with airfoil chord length $\left(C_{L}\right)$ and speed of sound $\left(c_{s}\right)$. The RANS data including the mean flow, turbulence kinetic energy $(k)$, integral length scales $(\Lambda)$ and integral time scales $(\tau)$ are mapped from the block-structured CFD mesh on the uniform RPM grid using ParaView. ${ }^{18}$ The mean flow and turbulence kinetic energy are directly obtained from the RANS computation. The integral length scales and time scales of turbulence are calculated using the turbulence kinetic energy and specific dissipation $\operatorname{rate}\left(\omega_{t}\right)$ as given in Appendix. 


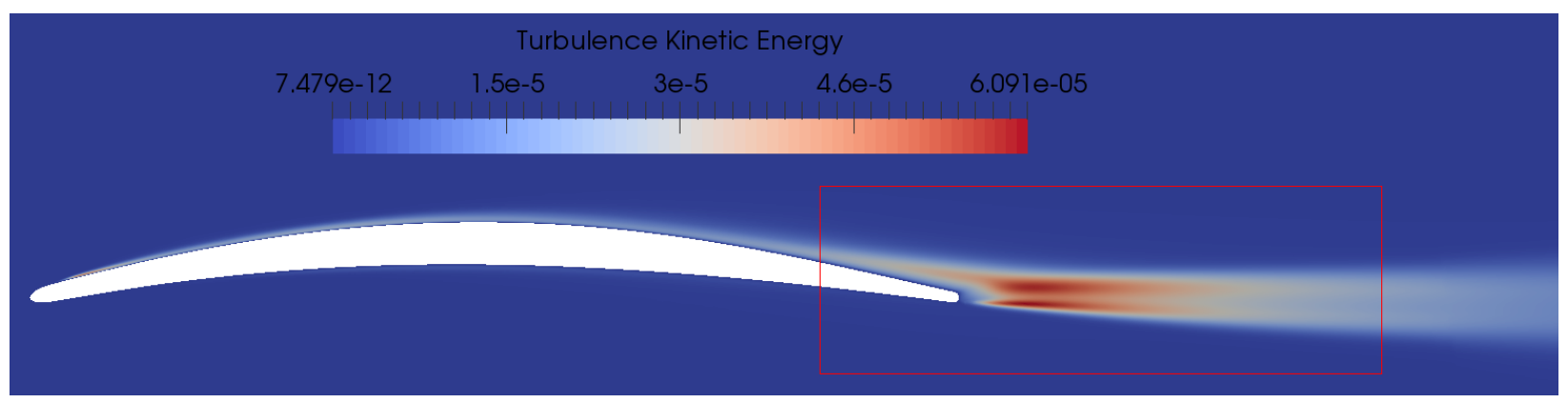

Figure 9. Airfoil rotated by the a.o.a $\alpha=8^{\circ}$ and the rectangular RPM domain(in red).

The quality of the synthetic turbulence generated by the stochastic method is assessed by comparing the statistics of the reconstructed fluctuations with the input RANS statistics. The statistical description of the generated fluctuations is sensitive to the input parameters viz RPM grid $\operatorname{size}\left(\Delta_{R P M}\right)$, no of particles $(N)$, sampling interval $\left(\Delta t_{s}\right)$ and length of the time signal $\left(T_{\text {end }}\right)$. The RPM grid $\operatorname{size}\left(\Delta_{R P M}\right)$ and no of particles $(N)$ are estimated based on the distribution of the integral length scale of turbulence. The sampling time $\left(\Delta t_{s}\right)$ is determined by the integral time scale of turbulence $(\tau)$. The length of the time signal $\left(T_{\text {end }}\right)$ required to accurately reproduce the RANS statistics is determined by the number of samples required for the convergence of the statistics. The simulation parameters of the stochastic method are listed in Table 1. Due to the stochastic nature of the method there is no definitive criteria for determining the simulation parameters. However, some preliminary best practice experience has been obtained for the application of RPM method to trailing-edge turbulence generation. ${ }^{19}$ The results of the computed statistical quantities for the choice of parameters listed in Table 1 are presented in this section.

Table 1. Choice of parameters for the stochastic method

\begin{tabular}{lc} 
Simulation Parameters & Non-dimensional Value \\
\hline Uniform RPM grid spacing $\left(\Delta_{R P M}\right)$ & 0.001 \\
No of particles $(N)$ & 120000 \\
Runge-Kutta time integration step $(\Delta t)$ & 0.002125 \\
Sampling interval $\left(\Delta t_{s}\right)$ & 0.02125 \\
Total time $\left(T_{\text {end }}\right)$ & 212.5 \\
No of samples $\left(N_{s}\right)$ & 10000
\end{tabular}

The comparison of the reconstructed turbulence kinetic energy with the RANS turbulence kinetic energy in domain in shown in Figure 10. The comparison along the $\mathrm{x}$-axis behind the trailing edge is shown in Figure 11. From the plots it is concluded that the variance of the reconstructed fluctuations closely reproduces the target variance.
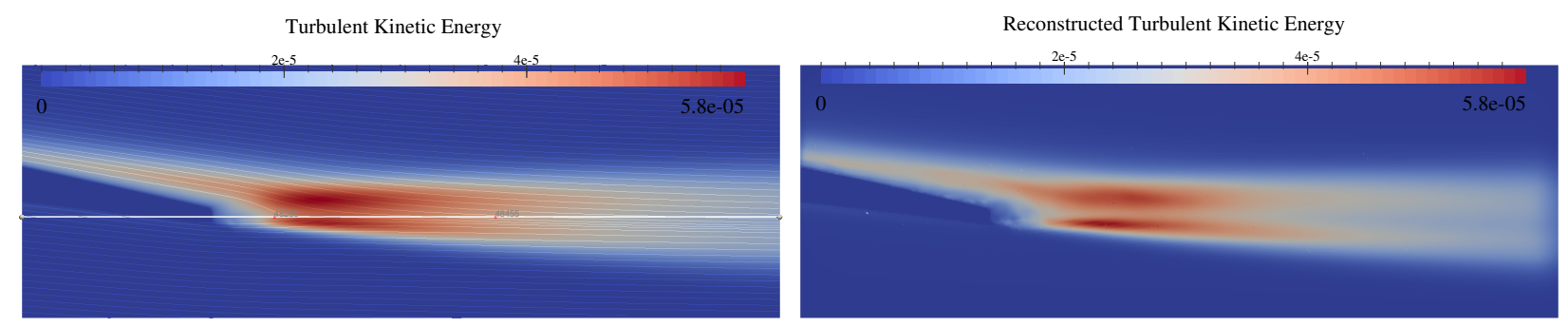

Figure 10. a) RANS turbulence kinetic Energy with reference points marked along the x-Axis. b) Reconstructed turbulence kinetic energy $K=\frac{1}{2}\left(\overline{u_{1}^{2}}+\overline{u_{2}^{2}}\right)$ 


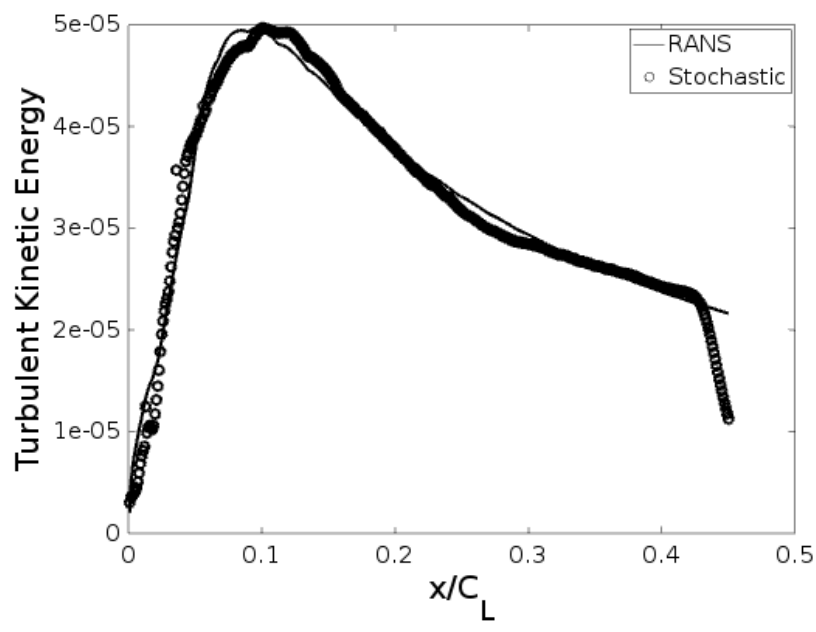

Figure 11. Reconstructed turbulence kinetic energy along the $\mathrm{x}$-Axis behind the trailing edge.

The statistical properties of the synthetic turbulence are further assessed by comparing numerical twopoint cross-correlations $R_{i j}(r, t)=<u_{i}\left(\overrightarrow{x_{1}}, t_{1}\right) u_{j}\left(\overrightarrow{x_{2}}, t_{2}\right)>$ where $r=\left|\overrightarrow{x_{2}}-\overrightarrow{x_{1}}\right|$ and $t=\left|t_{2}-t_{1}\right|$ with the analytical results. For a given model of the energy spectrum of turbulence it is possible to derive analytical expressions for the two-point cross-correlations $R_{i j}(r, t)$. For the Gaussian energy spectrum the analytical expressions for the two-point cross-correlations are given in Appendix. To check the correctness of the implementation of the stochastic reconstruction method the numerical two-point correlations in space and time are compared with the analytical expressions at arbitrary points in the domain. The results following the comparison along the $\mathrm{x}$-Axis are shown in Figure 12. From the plots it is concluded that the method is able to reproduce the two-point cross-correlations for the prescribed Gaussian filter and the exponential time decorrelation prescribed by the Langevin model.
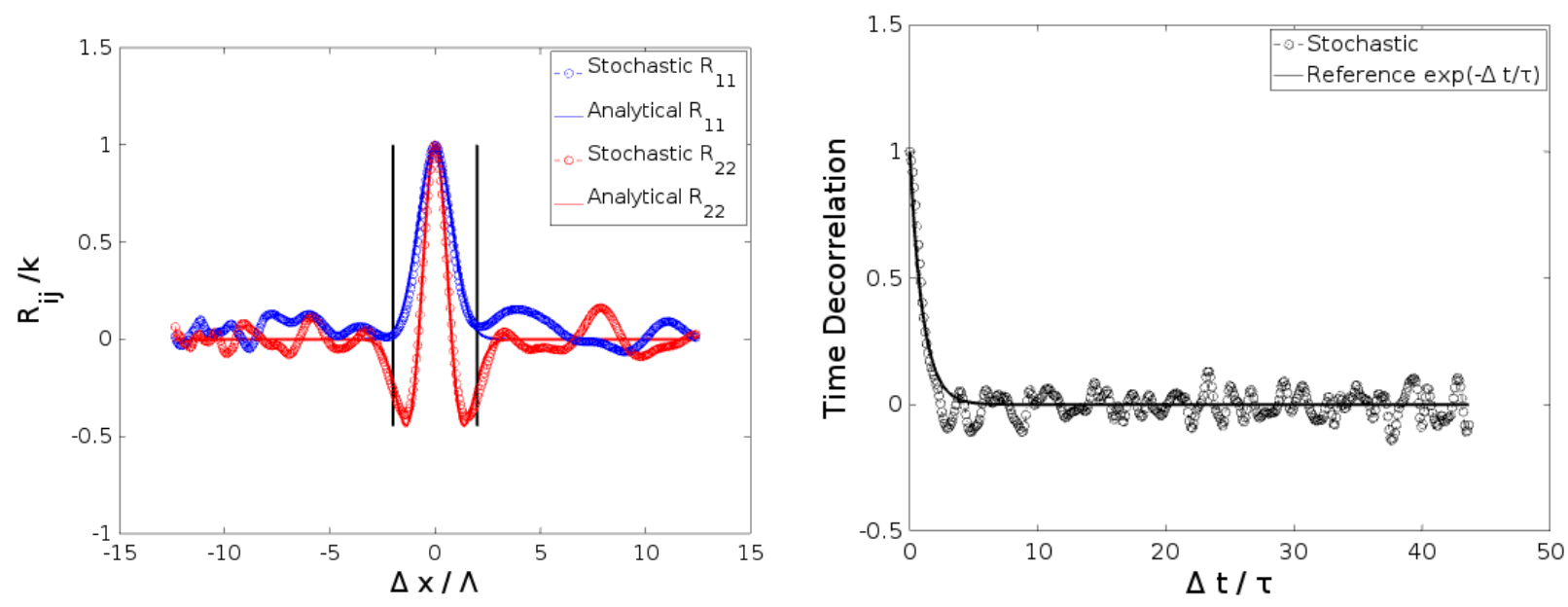

Figure 12. a) Correlation in space along x-Axis about mid reference point 48455 in Figure 10.a. b) Time correlation of a point moving with the mean flow along the streamline aligned with the $\mathrm{x}$-Axis with respect to the left reference point 48280 shown in Figure 10.a. 


\section{Acoustic Perturbation Equations}

The acoustic propagation is using the APE-4 system (for their formulation refer to Ewert et al. ${ }^{20}$ ) which for uniform steady mean density $\left(\rho^{0}\right)$ and $\operatorname{pressure}\left(p^{0}\right)$ can be written as

$$
\begin{gathered}
\frac{\partial \rho^{\prime}}{\partial t}+\nabla \cdot\left(\rho^{0} \overrightarrow{v^{\prime}}+\overrightarrow{U_{0}} \rho^{\prime}\right)=0, \\
\frac{\partial\left(\rho^{0} \overrightarrow{v^{\prime}}\right)}{\partial t}+\nabla\left(\overrightarrow{U_{0}} \cdot\left(\rho^{0} \overrightarrow{v^{\prime}}\right)\right)+c_{s}^{2} \nabla \rho^{\prime}=\rho^{0} \overrightarrow{L_{m}} .
\end{gathered}
$$

The fluctuating pressure can be obtained from density fluctuations using the homentropic relation $p^{\prime}=c_{s}^{2} \rho^{\prime}$. In the APE-4 system the fluctuating Lamb vector $\overrightarrow{L_{m}}$ occurs as a vortex sound source:

$$
\overrightarrow{L_{m}}=-(\vec{\omega} \times \vec{v})^{\prime}
$$

The Lamb vector can be approximated by discarding the non-linear terms as:

$$
\overrightarrow{L_{m}} \approx-\overrightarrow{\omega_{0}} \times \overrightarrow{v^{\prime}}-\overrightarrow{\omega^{\prime}} \times \overrightarrow{U_{0}}
$$

In the implementation of the stochastic method, the fluctuating vorticity $\vec{\omega}^{\prime}$ is obtained by taking the curl of the stochastically generated velocity fluctuations. The curl of the velocity fluctuations is conveniently calculated using second-order finite difference on the uniform RPM grid. The mean vorticity $\overrightarrow{\omega_{0}}$ and velocity $\vec{U}_{0}$ are obtained from RANS. Time snapshots of the generated fluctuating velocity field and the linearized Lamb vector are shown in Figure 13. To minimize the errors due to an abrupt truncation of the source region, ${ }^{21}$ the fluctuating Lamb vector $\overrightarrow{L_{m}}$ is windowed using a Gaussian function $e^{\frac{-x^{2}}{2 \sigma^{2} x_{D}{ }^{2}}}$ where $x_{D}$ is the width of the truncation boundaries on all the four sides as shown in Figure 13.b. This forces the sources to decay towards the four boundaries of the source domain minimizing the impact of spatial truncation errors.
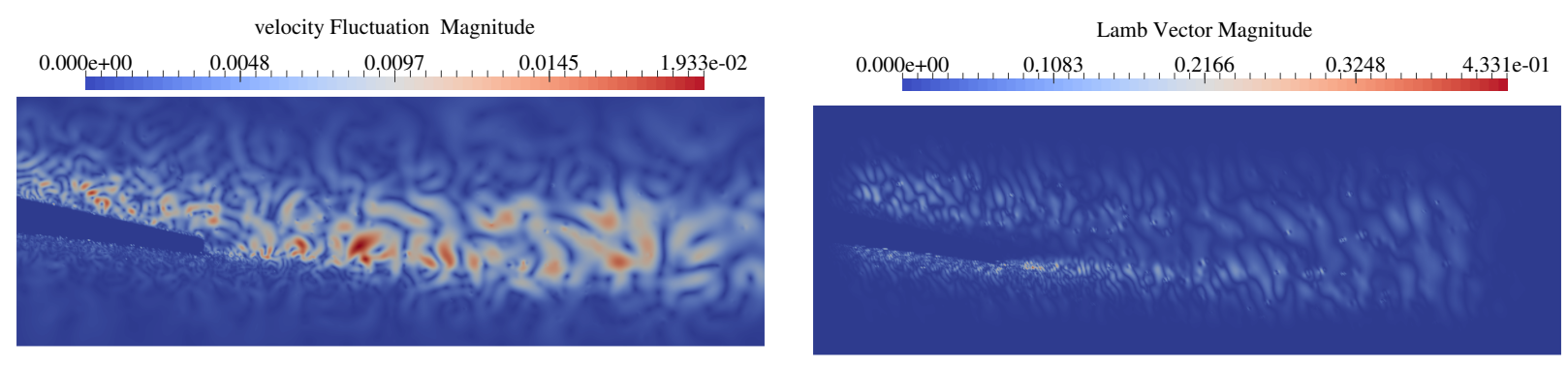

Figure 13. a) Snapshot of the magnitude of the fluctuating velocity vector at $\mathbf{t}=T_{\text {end }}$. b) Fluctuating Lamb vector magnitude at $\mathbf{t}=T_{\text {end }}$.

The APE-4 equations in the propagation domain are solved by a quadrature-free discontinuous Galerkin solver. ${ }^{17}$ The mean flow convection effects on the acoustic propagation are considered to be negligible due to the small Mach number Ma 0.05 based on free stream velocity. The unstructured DG Mesh around the CD airfoil is shown in Figure 14. The DG Mesh has 15840 triangular elements with increasing element size from 0.01 in the source region to 0.03 in the damping zone. The order of the polynomial basis functions for interpolation is chosen to be 6 , resulting into 28 nodes for every triangular element and total 32,134 nodes. Since the unstructured DG Mesh is coarse then the RPM mesh a conservative mapping scheme is used to transfer the source information from RPM mesh to DG mesh. The non-dimensional time step enforced by the CFL condition corresponding to the smallest element close to the trailing-edge is $\Delta t \frac{c_{s}}{C_{L}}=0.0006$. 


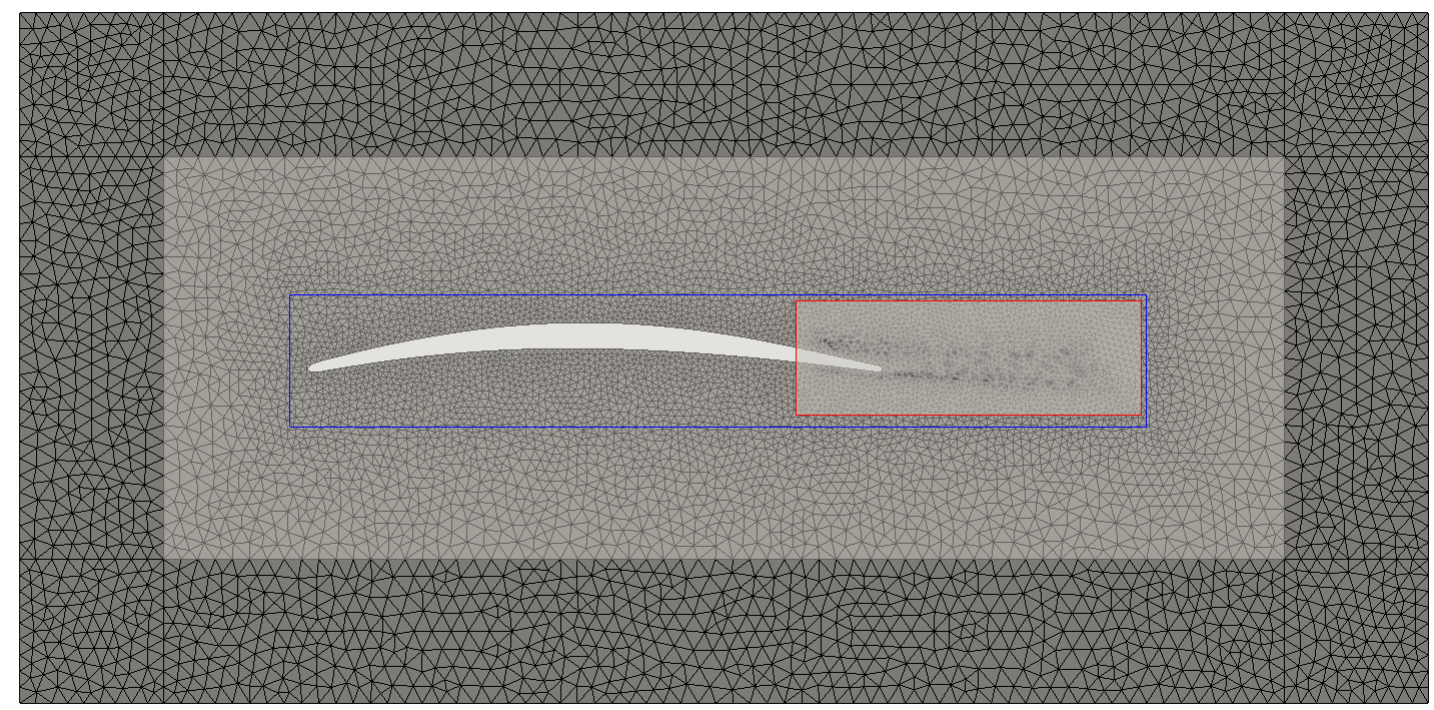

Figure 14. Unstructured DG Mesh with mesh refinement close to the airfoil and in the source region. The zone for damping the outgoing acoustic waves is highlighted in dark. The RPM domain is shown in red with the mapped source terms. The Kirchhoff surface is shown in blue.

\section{Kirchhoff extrapolation for far-field sound pressure level(SPL) prediction}

When the acoustic pressure in the mid field is known by solving the APE-4 system of equations, the Kirchhoff extrapolation method is used to obtain the far-field sound pressure levels. The Kirchhoff extrapolation method is a means of evaluating radiating sound in the far-field from flow acoustic quantities on a control surface put in the mid-field. The linear, homogeneous wave equation is assumed to be valid in the propagation region outside the Kirchhoff surface. The two dimensional frequency domain formulation of the Kirchhoff method $^{22}$ is given by

$$
\left.\hat{p^{\prime}}(\vec{x}, \kappa)=\oint_{S}\left[\frac{\partial \hat{p^{\prime}}}{\partial \eta}\left(\frac{i}{4} H_{0}{ }^{2}(\kappa r)\right)\right)-\hat{p}^{\prime} \cos (\theta)\left(\frac{i}{4} \kappa H_{1}{ }^{2}(\kappa r)\right)\right] d S,
$$

where $p^{\prime}$ is the acoustic pressure, $\kappa$ is the wave number, $r$ is the distance from the source to the observer's position, $\eta$ is the outward normal to the control surface and $\theta$ is the angle between $\vec{r}$ and $\vec{\eta}$. Here $H$ denotes the Hankel function and $\hat{\imath}$ denotes Fourier transform.

Since the propagation is in time domain, the time signal for the acoustic pressure and velocity fluctuations are extracted at the monitor points (shown in Figure 14) by interpolating from the neighbouring nodes of the acoustic mesh. The pressure and velocity data are then transformed to frequency domain using FFT. The gradient of the pressure fluctuations at the monitor points is obtained from the velocity fluctuations using the linearized inviscid momentum conservation equation written in frequency domain as:

$$
\nabla \hat{p^{\prime}}=i \omega\left(\rho_{0} \hat{\vec{v}^{\prime}}\right)
$$

\section{E. 2-D to 3-D correction}

The broadband predictions are based on $2 \mathrm{D}$ simulations for which the far-field acoustic pressure asymptotically decays with $p \propto 1 / \sqrt{(\kappa R)}$ instead of $p \propto 1 / R$. To correct for the artificial acoustic 2-D decay law taking into account the finite span of the airfoil a 2-D to 3-D correction methodology was introduced by Ewert et al. ${ }^{10}$ The $2 \mathrm{D}$ to $3 \mathrm{D}$ correction procedure is given by

$$
S P L_{3 D}=S P L_{2 D}+10 \log \left(\frac{C}{2 \pi} \frac{L}{R} M a\right)
$$

where $\mathrm{C}=1.4$ is a constant, $\mathrm{L}$ is the total span length of the airfoil, $\mathrm{R}$ is the far-field distance and Ma is the free-stream mach number of the flow. The correction preserves the shape of the $2 \mathrm{D}$ spectrum (i.e no frequency 
dependence) and the directivity(i.e. no $\theta$ dependence). The correction procedure stated above assumes that flow is statistically homogeneous in the spanwise direction with spanwise coherence length scale smaller than the wing span. For the CD Airfoil case the decay of the space-time correlations of the fluctuating pressure near the trailing-edge at $x_{c} / C=-0.02$ on the suction side and the corresponding spanwise coherence was reported in the work of Christophe et al. ${ }^{23}$ The correlation in the spanwise direction was found to be 0.1 at $\Delta z=0.02 L$. Therefore, the assumption of spanwise coherence length scale smaller than the wing span holds for the CD Airfoil case. Furthermore the far-field distance should be larger than the span length $(\mathrm{L}=0.3 \mathrm{~m})$ for the correction procedure. This also holds for the CD Airfoil case where the far-field SPL is compared with experimental data available at a distance of $R=2 m$ above the airfoil.

\section{Results}

The far-field SPL at a distance $R=2 \mathrm{~m}$ above the airfoil trailing-edge and at an angle $\theta=90^{\circ}$ with respect to the chord line is compared with the available experimental data in Figure 15. The numerical results have been processed to make them comparable with the experiments. The sound pressure level (SPL) is computed as follows

$$
S P L(\vec{x}, \omega)=10 \log \left(\frac{S_{p p}^{a v g}(\vec{x}, \omega) \Delta f^{e x p}}{p_{r e f}^{2}}\right)+10 \log \left(\frac{C}{2 \pi} \frac{L}{R} M a\right)
$$

where $p_{\text {ref }}=2 \times 10^{-5} \mathrm{~Hz}$ is the reference pressure and $S_{p p}^{a v g}$ is the average power spectral density (PSD) at the far-field location. The experimental data is integrated in bins of width $\Delta f^{e x p}=8 \mathrm{~Hz}$. The time signal for the fluctuating pressure and velocity field extracted on the Kirchhoff surface is divided into 7 time windows(with $50 \%$ overlap). The length of each time window is 25000 time steps with non-dimensional time step of size $\Delta t \frac{c_{s}}{C_{L}}=0.0006$. This yields a frequency resolution $\Delta f=167 \mathrm{~Hz}$. For Fourier transform of the time signal Hann window is applied on each segment. The average power spectral density is obtained by averaging the power spectral density's for each individual numerical solution obtained after Kirchhoff extrapolation. Since the acoustic source terms are stochastically generated the averaging is important to reduce the variance and to increase the degree of smoothness of the spectral estimate for trend prediction.

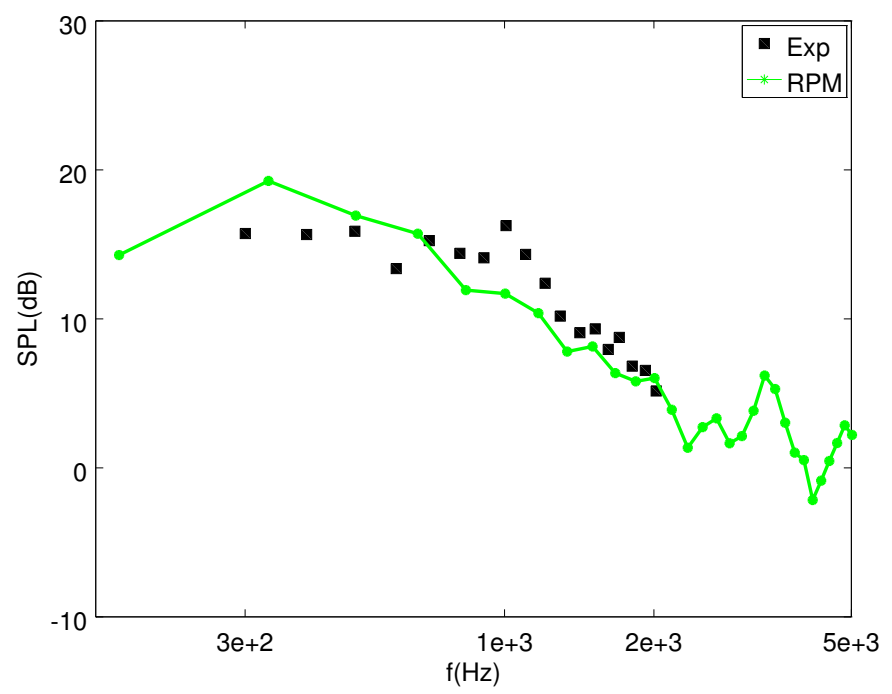

Figure 15. Far-field SPL at $\theta=90$, at a distance $R=2 \mathrm{~m}$ above the airfoil trailing-edge compared with experimental data.

The results are found to be in good agreement with experimental data within an error of $4.5 \mathrm{~dB}$ for the frequency range for which experimental data is available. 


\section{Conclusions}

In this work a hybrid RANS/CAA methodology in 2-D is applied to predict trailing-edge noise from a controlled-diffusion airfoil. The acoustic propagation is computed by solving the Acoustic Perturbation Equations, with vortex sound source terms. The acoustic source terms close to the trailing-edge are obtained from velocity fluctuations that are stochastically generated using the Random Particle Mesh method introduced by Ewert et al. The numerical results obtained are found to be in good agreement in the frequency range $300 \mathrm{~Hz}-2000 \mathrm{~Hz}$ for which experimental data is available. For higher frequencies $(>2500 \mathrm{~Hz})$ a gradual decay of the spectrum is observed contrary to a sharp decay of spectrum as observed in numerical studies reported in the literature. ${ }^{23,24}$ The decay of the spectrum observed for higher frequencies is not clear, and therefore needs further investigation. For design applications the 2-D CAA methodology as outlined in this work is found to be a promising alternative to expensive scale-resolved CFD in 3-D. In particular, this work demonstrates the applicability of the hybrid 2-D RANS/CAA approach for trailing-edge noise prediction from airfoils that have a uniform cross-section.

\section{Appendix}

The integral length scale $(\Lambda)$ and integral time scale $(\tau)$ of turbulence can be obtained from the turbulence kinetic $\operatorname{energy}(k)$ and specific dissipation $\operatorname{rate}\left(\omega_{t}\right)$ as:

$$
\begin{aligned}
\Lambda & =\frac{c_{l} \sqrt{k}}{C_{\mu} \omega_{t}}, \\
\tau & =\frac{2}{\beta C_{0} \omega_{t}},
\end{aligned}
$$

where $c_{l}=0.54, C_{\mu}=0.09, \beta=0.09$ and $C_{0}=2.1$ are model constants for the SST $k-\omega$ turbulence model. For 2-D homogeneous and isotropic turbulence analytical expressions for the spatial two-point cross-correlation tensor $R_{i j}$ can be obtained in terms of the longitudinal $f(r)$ and transverse $g(r)$ correlation functions as (see Pope $\left.^{25}\right)$ :

$$
R_{i j}(r)=k\left\{[f(r)-g(r)] \hat{r_{i}} \hat{r_{j}}+g(r) \delta_{i j}\right\} .
$$

The longitudinal and transverse correlation functions can be obtained from the correlation $C$ of the stream function $\eta$ as

$$
\begin{aligned}
& f(r)=\frac{-1}{r} \frac{d C(r)}{d r}, \\
& g(r)=-\frac{d^{2} C(r)}{d r^{2}},
\end{aligned}
$$

which for Gaussian energy spectrum is given by

$$
C(r)=\frac{2}{\pi} k \Lambda^{2} e^{\frac{-r^{2} \pi}{4 \Lambda^{2}}}
$$

where $r$ is the Lagrangian distance between the points.

\section{Acknowledgments}

The authors acknowledge with thanks the support of the European Commissions Framework Program Horizon 2020, through the Marie Skodowska-Curie Innovative Training Networks (ITN) AEOLUS4FUTURE

- Efficient harvesting of the wind energy (H2020-MSCA-ITN-2014: Grant agreement no. 643167) to the present research project.

\section{References}

\footnotetext{
${ }^{1}$ Caro, S. and Moreau, S., "Aeroacoustic modelling of low pressure axial flow fans," 6th Aeroacoustics Conference and Exhibit, 2000, p. 2094.

${ }^{2}$ Wang, M., Moreau, S., Iaccarino, G., and Roger, M., "LES prediction of wall-pressure fluctuations and noise of a low-speed airfoil," International journal of aeroacoustics, Vol. 8, No. 3, 2009, pp. 177-197.
} 
${ }^{3}$ Moreau, S., Neal, D., Khalighi, Y., Wang, M., and Iaccarino, G., "Validation of unstructured-mesh LES of the trailingedge flow and noise of a controlled-diffusion airfoil," Proceedings of the Summer Program, 2006, p. 1.

${ }^{4}$ Christophe, J., Anthoine, J., and Moreau, S., "Trailing edge noise of a controlled-diffusion airfoil at moderate and high angle of attack," 15th AIAA/CEAS Aeroacoustics Conference (30th AIAA Aeroacoustics Conference), p. 3196.

${ }^{5}$ Ewert, R. and Emunds, R., "CAA slat noise studies applying stochastic sound sources based on solenoidal digital filters," 11th AIAA/CEAS Aeroacoustics Conference, p. 2862.

${ }^{6}$ Ewert, R., "RPM-the fast Random Particle-Mesh method to realize unsteady turbulent sound sources and velocity fields for CAA applications," 13th AIAA/CEAS Aeroacoustics Conference (28th AIAA Aeroacoustics Conference), p. 3506.

${ }^{7}$ Siefert, M. and Ewert, R., "Anisotropic synthetic turbulence with sweeping generated by random particle-mesh method," Progress in Turbulence III, Springer, 2009, pp. 143-146.

${ }^{8}$ Ewert, R., "Broadband slat noise prediction based on CAA and stochastic sound sources from a fast random particle-mesh (RPM) method," Computers \& Fluids, Vol. 37, No. 4, 2008, pp. 369-387.

${ }^{9}$ Ewert, R., Dierke, J., Siebert, J., Neifeld, A., Appel, C., Siefert, M., and Kornow, O., "CAA broadband noise prediction for aeroacoustic design," Journal of sound and vibration, Vol. 330, No. 17, 2011, pp. 4139-4160.

${ }^{10}$ Ewert, R., Appel, C., Dierke, J., and Herr, M., "RANS/CAA based prediction of NACA 0012 broadband trailing edge noise and experimental validation," 15th AIAA/CEAS Aeroacoustics Conference (30th AIAA Aeroacoustics Conference), p. 3269.

${ }^{11}$ Vanelderen, B., De Roeck, W., and Desmet, W., "Flow noise prediction of confined flows using synthetic turbulence and linearized Euler equations in a hybrid methodology," 19th AIAA/CEAS Aeroacoustics Conference, 2013, p. 2267.

${ }^{12}$ Dieste, M., "Random-vortex-particle methods applied to broadband fan interaction noise," .

${ }^{13}$ Sté, Moreau, p., Henner, M., Iaccarino, G., Wang, M., and Roger, M., "Analysis of flow conditions in freejet experiments for studying airfoil self-noise," AIAA journal, Vol. 41, No. 10, 2003, pp. 1895-1905.

${ }^{14}$ Moreau, S., Neal, D., and Foss, J., "Hot-wire measurements around a controlled diffusion airfoil in an open-jet anechoic wind tunnel," Journal of Fluids Engineering, Vol. 128, No. 4, 2006, pp. 699-706.

${ }^{15}$ Weller, H. G., Tabor, G., Jasak, H., and Fureby, C., "A tensorial approach to computational continuum mechanics using object-oriented techniques," Computers in physics, Vol. 12, No. 6, 1998, pp. 620-631.

${ }^{16}$ Moreau, S. and Roger, M., "Effect of airfoil aerodynamic loading on trailing edge noise sources," AIAA journal, Vol. 43, No. 1, 2005, pp. 41-52

${ }^{17}$ Toulorge, T. and Desmet, W., "Curved boundary treatments for the discontinuous Galerkin method applied to aeroacoustic propagation," AIAA journal, Vol. 48, No. 2, 2010, pp. 479-489.

${ }^{18}$ Paraview, Paraview User Guide.

${ }^{19}$ Kadar, A., Martinez-Lera, P., Korchagin, V., De Roeck, W., and Desmet, W., "Stochastic reconstruction of turbulence for trailing-edge noise computation," 26th International Conference on Noise and Vibration engineering (ISMA2016), 2016.

${ }^{20}$ Ewert, R., Meinke, M., and Schroeder, W., "Computation of trailing edge noise of a 3d lifting airfoil in turbulent subsonic flow," 9th AIAA/CEAS Aeroacoustics Conference and Exhibit, p. 3114.

${ }^{21}$ Martínez-Lera, P. and Schram, C., "Correction techniques for the truncation of the source field in acoustic analogies," The Journal of the Acoustical Society of America, Vol. 124, No. 6, 2008, pp. 3421-3429.

${ }^{22}$ Scott, J., Pilon, A., Lyrintzis, A., Rozmajzl, T., Scott, J., Pilon, A., Lyrintzis, A., and Rozmajzl, T., "A numerical investigation of noise from a rectangular jet," 35th Aerospace Sciences Meeting and Exhibit, p. 285.

${ }^{23}$ Christophe, J., Anthoine, J. P., and Leduc, B., "Application of hybrid methods to high frequency aeroacoustics," 2011.

${ }^{24}$ Martnez-Lera, P., Christophe, J., and Schram, C., "Computation of the self-noise of a controlled-diffusion airfoil based on the acoustic analogy," International Journal of Aeroacoustics, Vol. 16, No. 1-2, 2017, pp. 44-64.

${ }^{25}$ Pope, S. B., "Turbulent flows," 2001. 